\title{
Necessary Conditions for Existence Results of Some Integral System
}

\author{
Yongxia Hua ${ }^{1}$ and Xiaohui $\mathrm{Yu}^{2}$ \\ ${ }^{1}$ Center of Applied Math, Harbin Institute of Technology, Shenzhen Graduate School, Shenzhen, Guangdong 518055, China \\ ${ }^{2}$ Institute for Advanced Study, Shenzhen University, Shenzhen, Guangdong 518060, China \\ Correspondence should be addressed to Xiaohui Yu; yuxiao_211@163.com
}

Received 3 May 2013; Accepted 13 July 2013

Academic Editor: Jaume Giné

Copyright (c) 2013 Y. Hua and X. Yu. This is an open access article distributed under the Creative Commons Attribution License, which permits unrestricted use, distribution, and reproduction in any medium, provided the original work is properly cited.

In this paper, we give some necessary conditions for the existence of positive solutions for integral systems.

\section{Introduction}

In this paper, we study the necessary condition for the existence of positive solutions for the following integral system:

$$
\begin{aligned}
& u(x)=\int_{\mathbb{R}^{N}}|x-y|^{\lambda} v(y)^{p} d y \text { in } \mathbb{R}^{N}, \\
& v(x)=\int_{\mathbb{R}^{N}}|x-y|^{\lambda} u(y)^{q} d y \quad \text { in } \mathbb{R}^{N},
\end{aligned}
$$

where $\lambda, p$, and $q$ are real parameters.

As for one single equation

$$
u(x)=\int_{\mathbb{R}^{N}}|x-y|^{\lambda} u(y)^{p} d y \quad \text { in } \mathbb{R}^{N}
$$

there are a lot of results of this problem. If $\lambda=\alpha-N$ with $0<\alpha<N$, then problem (2) is equivalent to the following differential equation:

$$
(-\Delta)^{\alpha / 2} u(x)=u(x)^{p} \text { in } \mathbb{R}^{N} .
$$

This problem has been widely studied in the past few years. For example, in order to answer a question raised by Lieb in [1], the authors studied the symmetric property and the uniqueness of solutions for problem (2) in [2]. Later, they studied the integral system (1) in [3]. Also, after the work of [2], Li studied the general form of (2) in [4]. For the case $\lambda<$ 0 , he obtained similar results to [2] but with less regularity requirement. For the case $\lambda>0$, he shows that if problem (2) has a nonnegative solution in $\mathbb{R}^{N}$ and $\lambda(p+1)+2 N \geq 0$, then $q=-1-2 N / \lambda$. The main ingredients in these papers are the moving plane method and moving sphere method based on the maximum principle of integral forms. This method has been widely used in other works. For example, inspired by these works, the author studied the Liouville-type theorems for problems (1) and (2) with general nonlinearities in $[5,6]$. For further results of this type of integral equations, see [718 , and so forth. We note that all these results concern the cases $\lambda<0$ and $p>0$. A natural question is whether similar results hold for $\lambda>0$ or $p<0$. We note that the case $\lambda<0$ and $p>0$ is quite different from the case $\lambda>0$ or $p<0$. Generally speaking, the moving plane method or the moving sphere method does not work in the latter case, so we have to look for other methods. In a recent paper [19], the author give a sufficient and necessary condition for the existence of positive solutions for problem (2) with $\lambda>0$. Based on some integral estimates, the author proved that problem (2) possesses a positive solution if and only if $\lambda p=-(\lambda+2 N)$. Inspired by the work of [19], we first study the integral system (1) with $\lambda>0$. Our main result is the following theorem.

Theorem 1. Suppose that $\lambda>0$ and problem (1) possesses $a C^{1}$ positive solution; then

$$
\frac{1}{1+p}+\frac{1}{1+q}=-\frac{\lambda}{N} .
$$

As for $\lambda<0$, we have the following nonexistence result. 
Theorem 2. If $-N<\lambda<0$, then problem (1) possesses no $C^{1}$ positive solution provided that $p<0$ or $q<0$.

This paper is organized as follows. We prove Theorem 1 in Section 2. The proof of Theorem 2 is completed in Section 3.

\section{Proof of Theorem 1}

We first claim that $u(x) \in L^{q+1}\left(\mathbb{R}^{N}\right)$ and $v(x) \in L^{p+1}\left(\mathbb{R}^{N}\right)$.

In fact, we infer from

$$
\begin{aligned}
v(0) & =\int_{\mathbb{R}^{N}}|y|^{\lambda} u(y)^{q} d y \\
& \geq \int_{\mathbb{R}^{N} \backslash B_{1}(0)}|y|^{\lambda} u(y)^{q} d y \\
& \geq \int_{\mathbb{R}^{N} \backslash B_{1}(0)} u(y)^{q} d y
\end{aligned}
$$

that $u(x) \in L^{q}\left(\mathbb{R}^{N}\right)$. Also, it follows from (1) that

$$
\begin{aligned}
|x|^{\lambda} u\left(\frac{x}{|x|^{2}}\right) & =\int_{\mathbb{R}^{N}}|x|^{\lambda}\left|\frac{x}{|x|^{2}}-y\right|^{\lambda} v(y)^{p} d y \\
& =\int_{\mathbb{R}^{N}}|y|^{\lambda}\left|x-\frac{y}{|y|^{2}}\right|^{\lambda} v^{p}(y) d y .
\end{aligned}
$$

Now taking limit in (6) by letting $x \rightarrow 0$, we obtain

$$
\lim _{|x| \rightarrow 0}\left[|x|^{\lambda} u\left(\frac{x}{|x|^{2}}\right)\right]=\int_{\mathbb{R}^{N}} v^{p}(y) d y<\infty .
$$

We point out that we can take the limit under the integral sign because of the dominated convergence theorem. In fact, we note that when $\lambda>0$ and $|x| \leq 1$, we have

$$
\left|x-\frac{y}{|y|^{2}}\right|^{\lambda} \leq\left(|x|+\frac{1}{|y|}\right)^{\lambda} \leq\left(1+\frac{1}{|y|}\right)^{\lambda} .
$$

It is easy to check that $|y|^{\lambda}(1+1 /|y|)^{\lambda} v^{p}(y) \in L^{1}\left(\mathbb{R}^{N}\right)$.

It follows from (7) that there exist $R>0$ and $C>0$ such that

$$
C^{-1}|x|^{\lambda} \leq u(x) \leq C|x|^{\lambda}
$$

for $|x| \geq R$. Finally, we have

$$
\begin{aligned}
\int_{\mathbb{R}^{N} \backslash B_{R}(0)} u^{q+1}(x) d x & =\int_{\mathbb{R}^{N} \backslash B_{R}(0)} u^{q} u d x \\
& \leq C \int_{\mathbb{R}^{N}}|x|^{\lambda} u^{q} d x \\
& =C v(0),
\end{aligned}
$$

which further implies that $u(x) \in L^{q+1}\left(\mathbb{R}^{N}\right)$. Similarly, we have $v(x) \in L^{p+1}\left(\mathbb{R}^{N}\right)$.
Next, we can prove as in [19] that

$$
\begin{aligned}
& \nabla u(x)=\lambda \int_{\mathbb{R}^{N}}|x-y|^{\lambda-2}(x-y) v^{p}(y) d y, \\
& \nabla v(x)=\lambda \int_{\mathbb{R}^{N}}|x-y|^{\lambda-2}(x-y) u^{q}(y) d y
\end{aligned}
$$

in the sense of distribution. Hence, we infer from (11) that

$$
\begin{aligned}
\nabla u^{1+q}(x)= & (1+q) u^{q}(x) \lambda \\
& \cdot \int_{\mathbb{R}^{N}}|x-y|^{\lambda-2}(x-y) v^{p}(y) d y, \\
\nabla v^{1+p}(x)= & (1+p) v^{p}(x) \lambda \\
& \cdot \int_{\mathbb{R}^{N}}|x-y|^{\lambda-2}(x-y) u^{q}(y) d y .
\end{aligned}
$$

Now we choose a cut-off function $\eta \in C^{\infty}([0,+\infty))$ satisfying $0 \leq \eta \leq 1,0 \leq\left|\eta^{\prime}\right| \leq 2, \eta(t)=1$ for $t \leq 1$ and $\eta(t)=0$ for $t \geq 2$. For any $R>0$, if we multiply (12) by $\eta(|x| / R) x$ and integrate over $\mathbb{R}^{N}$, then we get

$$
\begin{aligned}
& \int_{\mathbb{R}^{N}} \eta\left(\frac{|x|}{R}\right) v^{p}(x)\langle x, \nabla v(x)\rangle d x \\
& \quad=\int_{\mathbb{R}^{N}} \eta\left(\frac{|x|}{R}\right) v^{p} \int_{\mathbb{R}^{N}} \lambda|x-y|^{\lambda-2}\langle x, x-y\rangle u(y)^{q} d y d x,
\end{aligned}
$$

$$
\begin{aligned}
& \int_{\mathbb{R}^{N}} \eta\left(\frac{|x|}{R}\right) u^{q}(x)\langle x, \nabla u(x)\rangle d x \\
& =\int_{\mathbb{R}^{N}} \eta\left(\frac{|x|}{R}\right) u^{q}(x) \int_{\mathbb{R}^{N}} \lambda|x-y|^{\lambda-2}\langle x, x-y\rangle v(y)^{p} d y d x .
\end{aligned}
$$

While the left-hand side of (13) equals

$$
\begin{aligned}
\int_{\mathbb{R}^{N}} \eta\left(\frac{|x|}{R}\right) v^{p}(x)\langle x, \nabla v(x)\rangle d x \\
=\frac{1}{1+p} \int_{\mathbb{R}^{N}} \eta\left(\frac{|x|}{R}\right)\left\langle x, \nabla v^{1+p}(x)\right\rangle d x \\
=-\frac{N}{1+p} \int_{\mathbb{R}^{N}} \eta\left(\frac{|x|}{R}\right) v^{1+p}(x) d x \\
-\frac{1}{1+p} \int_{\mathbb{R}^{N}}\left\langle\nabla\left(\eta\left(\frac{|x|}{R}\right)\right), x\right\rangle v^{1+p} d x,
\end{aligned}
$$

it follows from

$$
\left\langle\nabla\left(\eta\left(\frac{|x|}{R}\right)\right), x\right\rangle \leq \frac{2|x|}{R}
$$

that

$$
\begin{gathered}
\int_{\mathbb{R}^{N}}\left\langle\nabla\left(\eta\left(\frac{|x|}{R}\right)\right), x\right\rangle v^{1+p} d x \\
\leq 4 \int_{R \leq|x| \leq 2 R} v^{1+p} d x \longrightarrow 0
\end{gathered}
$$


as $R \rightarrow \infty$ by $v \in L^{1+p}\left(\mathbb{R}^{N}\right)$. Thus we conclude that

$$
\begin{aligned}
& \lim _{R \rightarrow \infty} \int_{\mathbb{R}^{N}} \eta\left(\frac{|x|}{R}\right) v^{p}(x)\langle x, \nabla v(x)\rangle d x \\
& =-\frac{N}{1+p} \int_{\mathbb{R}^{N}} v^{1+p}(x) d x .
\end{aligned}
$$

While the right-hand side of (13) equals

$$
\begin{aligned}
& \lambda \int_{\mathbb{R}^{N}} \eta\left(\frac{|x|}{R}\right) v^{p}(x) \\
& \cdot \int_{\mathbb{R}^{N}}|x-y|^{\lambda-2}\langle x, x-y\rangle u(y)^{q} d y d x \\
& =\frac{\lambda}{2} \int_{\mathbb{R}^{N}} \eta\left(\frac{|x|}{R}\right) v^{p}(x) \\
& \cdot \int_{\mathbb{R}^{N}}|x-y|^{\lambda-2}\langle x-y, x-y\rangle u(y)^{q} d y d x \\
& +\frac{\lambda}{2} \int_{\mathbb{R}^{N}} \eta\left(\frac{|x|}{R}\right) v^{p}(x) \\
& \cdot \int_{\mathbb{R}^{N}}|x-y|^{\lambda-2}\langle x+y, x-y\rangle u(y)^{q} d y d x \\
& =\frac{\lambda}{2} \int_{\mathbb{R}^{N}} \eta\left(\frac{|x|}{R}\right) v^{1+p}(x) d x \\
& +\frac{\lambda}{2} \int_{\mathbb{R}^{N}} \eta\left(\frac{|x|}{R}\right) v^{p}(x) \\
& \cdot \int_{\mathbb{R}^{N}}|x-y|^{\lambda-2}\langle x+y, x-y\rangle u(y)^{q} d y d x,
\end{aligned}
$$

it can be checked as in [19] that

$$
\int_{\mathbb{R}^{N}} \int_{\mathbb{R}^{N}}|x-y|^{\lambda-2}\langle x+y, x-y\rangle u(y)^{q} v(x)^{p} d y d x<\infty .
$$

Hence, by letting $R \rightarrow \infty$ in (19) we get

$$
\begin{aligned}
\lim _{R \rightarrow \infty} \lambda \int_{\mathbb{R}^{N}} \eta\left(\frac{|x|}{R}\right) v^{p}(x) & \cdot \int_{\mathbb{R}^{N}}|x-y|^{\lambda-2}\langle x, x-y\rangle u(y)^{q} d y d x \\
= & \frac{\lambda}{2} \int_{\mathbb{R}^{N}} v^{1+p}(x) d x+\frac{\lambda}{2} \\
\cdot \int_{\mathbb{R}^{N}} \int_{\mathbb{R}^{N}}|x-y|^{\lambda-2} & \cdot\langle x+y, x-y\rangle u^{q}(y) v^{p}(x) d y d x .
\end{aligned}
$$

We infer from (13), (18), and (21) that

$$
\begin{aligned}
& -\frac{N}{1+p} \int_{\mathbb{R}^{N}} v^{1+p}(x) d x \\
& =\frac{\lambda}{2} \int_{\mathbb{R}^{N}} v^{1+p}(x) d x \\
& \quad+\frac{\lambda}{2} \int_{\mathbb{R}^{N}} \int_{\mathbb{R}^{N}}|x-y|^{\lambda-2} \\
& \quad \cdot\langle x+y, x-y\rangle u^{q}(y) v^{p}(x) d y d x .
\end{aligned}
$$

Similarly, we can prove that

$$
\begin{aligned}
& -\frac{N}{1+p} \int_{\mathbb{R}^{N}} u^{1+q}(x) d x \\
& =\frac{\lambda}{2} \int_{\mathbb{R}^{N}} u^{1+q}(x) d x \\
& \quad+\frac{\lambda}{2} \int_{\mathbb{R}^{N}} \int_{\mathbb{R}^{N}}|x-y|^{\lambda-2} \\
& =\frac{\lambda}{2} \int_{\mathbb{R}^{N}} u^{1+q}(x) d x \\
& \quad-\frac{\lambda}{2} \int_{\mathbb{R}^{N}} \int_{\mathbb{R}^{N}}|x-y|^{\lambda-2} \\
& \quad \cdot\langle x+y, x-y\rangle u^{q}(x) v^{p}(y) d y d x
\end{aligned}
$$

The above two equations imply that

$$
\begin{gathered}
-\frac{N}{1+p} \int_{\mathbb{R}^{N}} u^{1+q}(x) d x-\frac{N}{1+p} \int_{\mathbb{R}^{N}} v^{1+p}(x) d x \\
=\frac{\lambda}{2}\left[\int_{\mathbb{R}^{N}} u^{1+q}(x) d x+\int_{\mathbb{R}^{N}} v^{1+p}(x) d x\right] .
\end{gathered}
$$

On the other hand, since

$$
v(x)^{1+p}=v(x)^{p} \int_{\mathbb{R}^{N}}|x-y|^{\lambda} u^{q}(y) d y,
$$

we have

$$
\begin{aligned}
\int_{\mathbb{R}^{N}} v^{1+p}(x) d x \\
\quad=\int_{\mathbb{R}^{N}} \int_{\mathbb{R}^{N}}|x-y|^{\lambda} u^{q}(y) v(x)^{p} d y d x
\end{aligned}
$$

by taking into account that $v(x) \in L^{1+p}\left(\mathbb{R}^{N}\right)$. Similarly, we have

$$
\begin{aligned}
& \int_{\mathbb{R}^{N}} u^{1+q}(x) d x \\
& =\int_{\mathbb{R}^{N}} \int_{\mathbb{R}^{N}}|x-y|^{\lambda} u^{q}(y) v(x)^{p} d y d x .
\end{aligned}
$$


Then it follows from (26) and (27) that

$$
\int_{\mathbb{R}^{N}} u^{1+q}(x) d x=\int_{\mathbb{R}^{N}} v^{1+p}(x) d x
$$

Finally, we infer from (24) and (28) that

$$
\frac{1}{1+p}+\frac{1}{1+q}=-\frac{\lambda}{N}
$$

This completes the proof of Theorem 1 .

\section{Proof of Theorem 2}

We assume that $q<0$ without loss of generality. First, we note that by Lemma 3.11.3 in [20], we have, for all $r>0$,

$$
\begin{aligned}
& \frac{1}{\omega_{N} r^{N}} \int_{B_{r}(0)} u(x) d x \\
& \quad=\int_{\mathbb{R}^{N}}\left\{\frac{1}{\omega_{N} r^{N}} \int_{B_{r}(0)}|x-y|^{\lambda} d x\right\} v^{p}(y) d y \\
& \quad \leq C \int_{\mathbb{R}^{N}}|y|^{\lambda} v^{p}(y) d y=C u(0) .
\end{aligned}
$$

Similarly, we have

$$
\frac{1}{\omega_{N} r^{N}} \int_{B_{r}(0)} v(x) d x \leq C v(0)
$$

for any $r>0$.

If we choose $\alpha=q /(q-1), \beta=1-q$, and $\delta=(q-1) / q$, then we can infer from the Holder inequality that

$$
\begin{aligned}
1= & \frac{1}{\omega_{N} r^{N}} \int_{B_{r}(0)} u(x)^{-\alpha} u(x)^{\alpha} d x \\
\leq & \left(\frac{1}{\omega_{N} r^{N}} \int_{B_{r}(0)} u(x)^{-\alpha \beta} d x\right)^{1 / \beta} \\
& \cdot\left(\frac{1}{\omega_{N} r^{N}} \int_{B_{r}(0)} u(x)^{\alpha \delta} d x\right)^{1 / \delta} \\
= & \left(\frac{1}{\omega_{N} r^{N}} \int_{B_{r}(0)} u(x)^{q} d x\right)^{1 /(1-q)} \\
& \cdot(C u(0))^{q /(q-1)} .
\end{aligned}
$$

That is,

$$
(C u(0))^{q} \leq \frac{1}{\omega_{N} r^{N}} \int_{B_{r}(0)} u^{q}(x) d x
$$

Since $\lambda<0$, so if $|x|<r$, then we have $r^{\lambda}<|x|^{\lambda}$. Multiplying both sides of (33) by $\omega_{N} r^{N+\lambda}$, we get

$$
\begin{aligned}
& C^{q} \omega_{N} r^{N+\lambda} u(0)^{q} \\
& \quad \leq \omega_{N} r^{N+\lambda} \frac{1}{\omega_{N} r^{N}} \int_{B_{r}(0)} u^{q}(x) d x \\
& \quad=r^{\lambda} \int_{B_{r}(0)} u^{q}(x) d x \\
& \quad \leq \int_{B_{r}(0)}|x|^{\lambda} u^{q}(x) d x=v(0) .
\end{aligned}
$$

Since $-N<\lambda<0$, we have $N+\lambda>0$. Hence the left-hand side of (34) goes to infinity as $r \rightarrow \infty$, which is a contradiction. This completes the proof.

\section{Acknowledgments}

The first author is supported by SZPP (no. KQCX20120802140634893), Guangdong S\&T Major Project (no. 2012A080104014), and SRF for ROCS, SEM. Xiaohui Yu is supported by NSFC no. 11101291. The authors would like to thank the anonymous referees for their valuable suggestions.

\section{References}

[1] E. H. Lieb, "Sharp constants in the Hardy-Littlewood-Sobolev and related inequalities," Annals of Mathematics. Second Series, vol. 118, no. 2, pp. 349-374, 1983.

[2] W. Chen, C. Li, and B. Ou, "Classification of solutions for an integral equation," Communications on Pure and Applied Mathematics, vol. 59, no. 3, pp. 330-343, 2006.

[3] W. Chen, C. Li, and B. Ou, "Classification of solutions for a system of integral equations," Communications in Partial Differential Equations, vol. 30, no. 1-3, pp. 59-65, 2005.

[4] Y. Y. Li, "Remark on some conformally invariant integral equations: the method of moving spheres," Journal of the European Mathematical Society, vol. 6, no. 2, pp. 153-180, 2004.

[5] X. Yu, "Liouville type theorems for integral equations and integral systems," Calculus of Variations and Partial Differential Equations, vol. 46, no. 1-2, pp. 75-95, 2013.

[6] X. Yu, "Liouville type theorems for singular integral equations and integral systems," Calculus of Variations and Partial Differential Equations, vol. 46, no. 1-2, pp. 75-95, 2013.

[7] W. Chen and C. Li, "An integral system and the Lane-Emden conjecture," Discrete and Continuous Dynamical Systems. Series A, vol. 24, no. 4, pp. 1167-1184, 2009.

[8] W. Chen and C. Li, "Classification of positive solutions for nonlinear differential and integral systems with critical exponents," Acta Mathematica Scientia. Series B. English Edition, vol. 29, no. 4, pp. 949-960, 2009.

[9] W. Chen and C. Li, Methods on Nonlinear Elliptic Equations, vol. 4 of AIMS Series on Differential Equations \& Dynamical Systems, American Institute of Mathematical Sciences, Springfield, Mo, USA, 2010

[10] W. Chen and C. Li, "Radial symmetry of solutions for some integral systems of Wolff type," Discrete and Continuous Dynamical Systems. Series A, vol. 30, no. 4, pp. 1083-1093, 2011. 
[11] F. Hang, X. Wang, and X. Yan, "An integral equation in conformal geometry," Annales de l'Institut Henri Poincaré. Analyse Non Linéaire, vol. 26, no. 1, pp. 1-21, 2009.

[12] C. Jin and C. Li, "Symmetry of solutions to some systems of integral equations," Proceedings of the American Mathematical Society, vol. 134, no. 6, pp. 1661-1670, 2006.

[13] L. Ma and D. Chen, "A Liouville type theorem for an integral system," Communications on Pure and Applied Analysis, vol. 5, no. 4, pp. 855-859, 2006.

[14] L. Ma and D. Chen, "Radial symmetry and monotonicity for an integral equation," Journal of Mathematical Analysis and Applications, vol. 342, no. 2, pp. 943-949, 2008.

[15] L. Ma and D. Chen, "Radial symmetry and uniqueness for positive solutions of a Schrödinger type system," Mathematical and Computer Modelling, vol. 49, no. 1-2, pp. 379-385, 2009.

[16] C. Ma, W. Chen, and C. Li, "Regularity of solutions for an integral system of Wolff type," Advances in Mathematics, vol. 226, no. 3, pp. 2676-2699, 2011.

[17] X. Xu, "Exact solutions of nonlinear conformally invariant integral equations in $R^{3}$," Advances in Mathematics, vol. 194, no. 2, pp. 485-503, 2005.

[18] X. Xu, "Uniqueness and non-existence theorems for conformally invariant equations," Journal of Functional Analysis, vol. 222, no. 1, pp. 1-28, 2005.

[19] X. Xu, "Uniqueness theorem for integral equations and its application," Journal of Functional Analysis, vol. 247, no. 1, pp. 95-109, 2007.

[20] W. P. Ziemer, Weakly Differentiable Functions. Sobolev Spaces and Functions of Bounded Variation, vol. 120 of Graduate Texts in Mathematics, Springer, New York, NY, USA, 1989. 


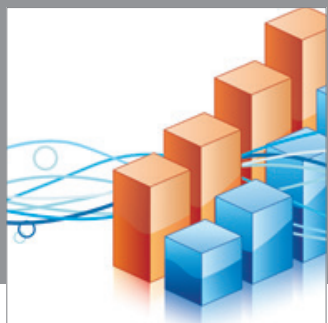

Advances in

Operations Research

mansans

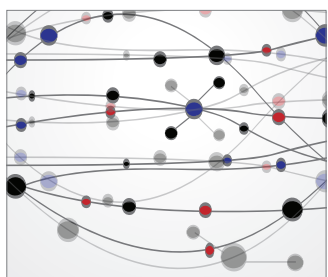

The Scientific World Journal
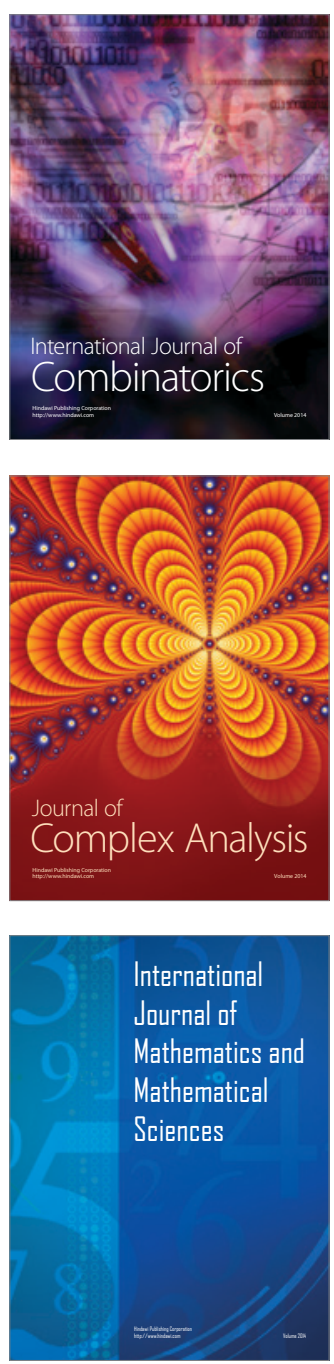
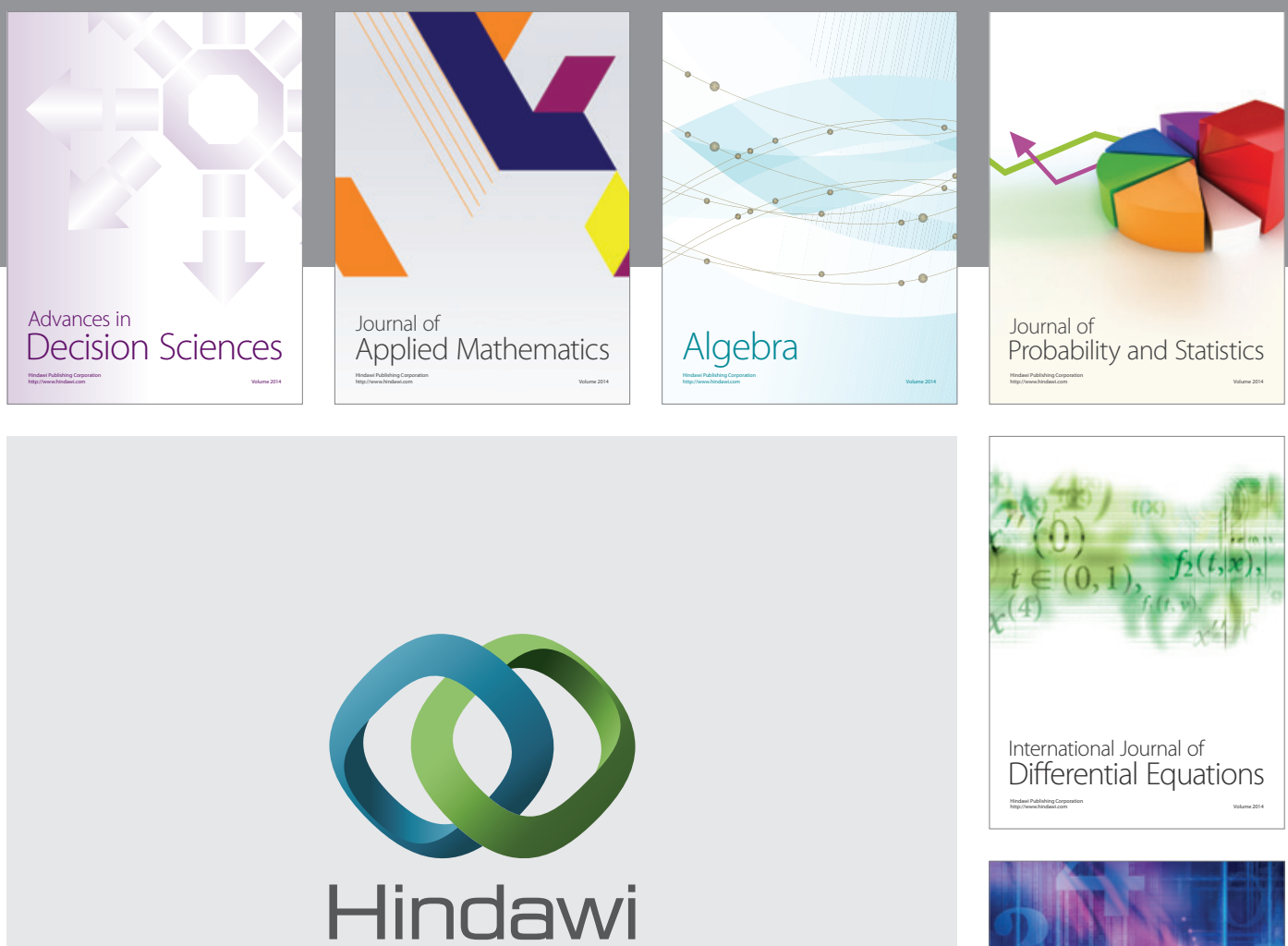

Submit your manuscripts at http://www.hindawi.com
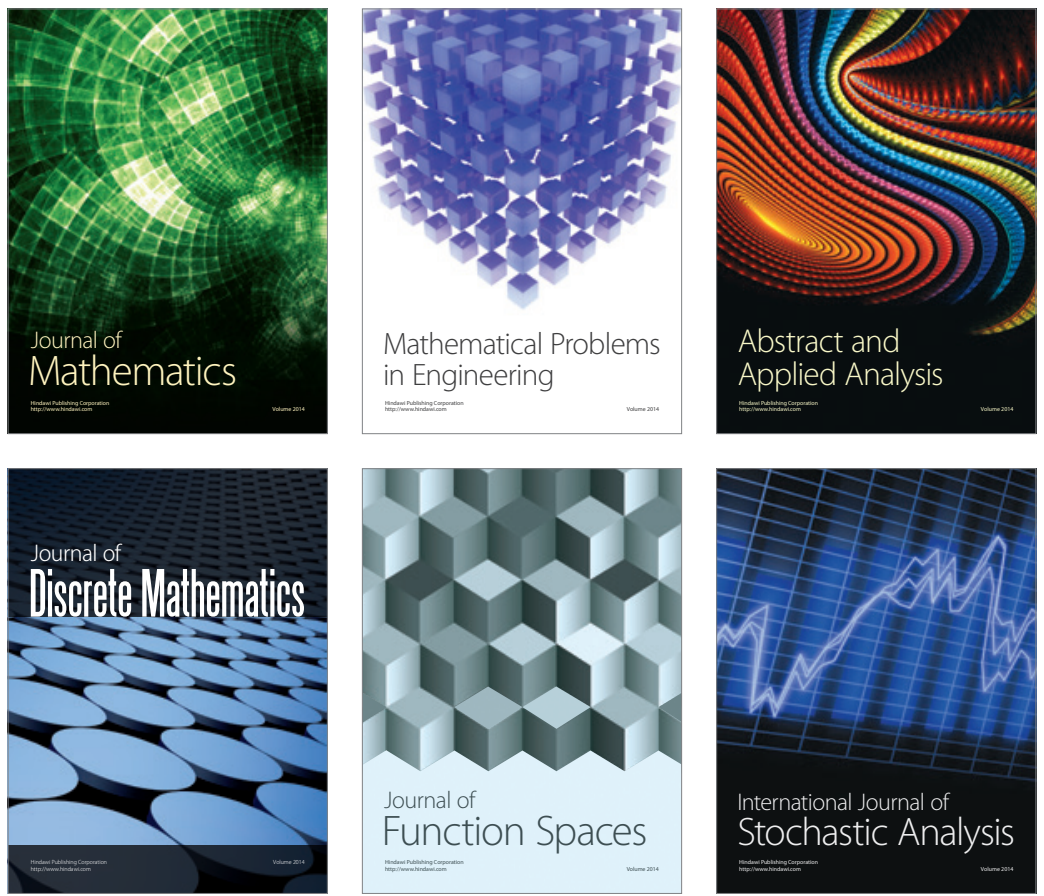

Journal of

Function Spaces

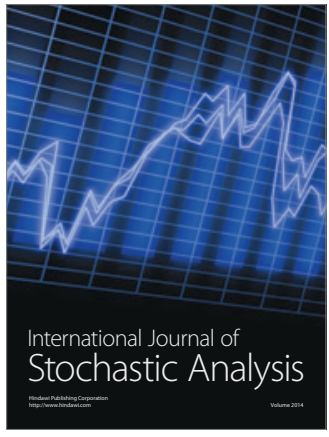

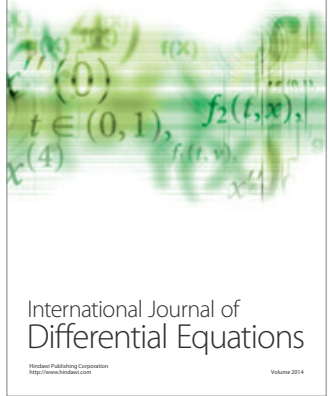
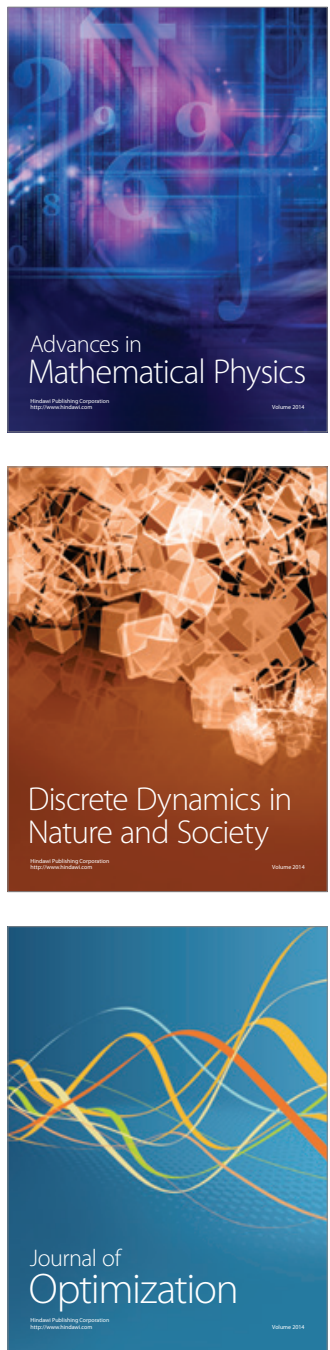